\title{
Graphene-based LbL deposited films: further study of electrical and gas sensing properties
}

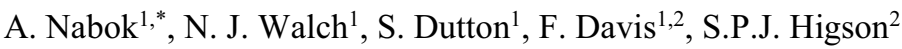 \\ ${ }^{1}$ Sheffield Hallam University, Materials and Engineering Research Institute, Sheffield, S11 7JN, UK \\ 2 Department of Engineering and Applied Design, University of Chichester, PO21 1HR, UK
}

\begin{abstract}
Graphene-surfactant composite materials obtained by the ultrasonic exfoliation of graphite powder in the presence of ionic surfactants (either CTAB or SDS) were utilised to construct thin films using layer-bylayer (LbL) electrostatic deposition technique. A series of graphene-based thin films were made by alternating layers of either graphene-SDS with polycations (PEI or PAH) or graphene-CTAB with polyanions (PSS). Also, graphene-phthalocyanine composite films were produced by alternating layers of graphene-CTAB with tetrasulfonated nickel phthalocyanine. Graphene-surfactant LbL films exhibited good electric conductivity (about $0.1 \mathrm{~S} / \mathrm{cm}$ ) of semiconductor type with a band gap of about $20 \mathrm{meV}$. Judging from UV-vis spectra measurements, graphene-phthalocyanine LbL films appeared to form joint $\pi$-electron system. Gas sensing testing of such composite films combining high conductivity of graphene with the gas sensing abilities of phthalocyanines showed substantial changes (up to 10\%) in electrical conductivity upon exposure to electroactive gases such as $\mathrm{HCl}$ and $\mathrm{NH}_{3}$.
\end{abstract}

\section{Introduction}

Graphene is one of the most popular materials of modern times with thousands of publications covering all possible aspects of the physical-chemical properties of graphenebased materials and their applications. Among several known technologies of graphene production, the method of ultrasonic exfoliation of graphite powder in the presence of surfactants $[1,2]$ is perhaps the least expensive. The resulting products, i.e. graphenesurfactant composite materials, have the useful properties of solubility in water and some organic solvents, thus enabling deposition by the simple thin film technologies of Langmuir-Blodgett (LB), spin-coating, and electrostatic layer-by-layer deposition [2, 3]. The electric properties of such films must be different from those of pristine graphene exhibiting high conductivity in the range of $10^{6} \mathrm{~S} / \mathrm{cm}$ caused by the large conjugated system of $\pi$-electrons with a zero band gap $[4,5]$. Our previous study of LB and LbL graphene-surfactant composite films showed high electric conductivity and a temperature dependence of conductivity of semiconductor type (conductivity rising with temperature), though that required further verification.

The use of electrostatic LbL deposition offer another attractive possibility of alternating electrically charged graphene layers with other molecular layers, for example phthalocyanines. It is known that graphene itself is not ideal for gas sensing and requires functionalization with other molecular receptors to achieve the required gas sensitivity and selectivity [6, 7]. On the other hand, metal-phthalocyanines thin films are known by excellent gas sensitivity to electro-active gases though their conductivity is very poor [8]. A combination of highly conductive graphene with metal-phthalocyanines seems to be very attractive for development of novel gas sensors.

In the current research we continue studying electric conductivity of graphene-surfactant composite films with particular focus in temperature dependence of conductivity. The second part of this work is dedicated to making graphene-phthalocyanine LbL films and studying their optical and electrical properties including the effect of electro-active gases.

\section{Experimental methodology}

\subsection{Samples preparation}

Graphene composites were prepared by a multistage sonication process of graphite powder in the presence of ionic surfactants containing either negative or positive ionic groups, e.g. sodium dodecyl sulphate (SDS) and cetyl- trimethyl- ammonium bromide (CTAB), as described earlier $[2,3]$. The resulted two graphenesurfactant composite materials, namely graphene-CTAB, and graphene-SDS (see schematic diagrams in figure 1a, b) appeared to be soluble in both water and some organic solvents, such as chloroform, and thus suitable for both

\footnotetext{
* Corresponding author: A.Nabok@,shu.ac.uk
} 


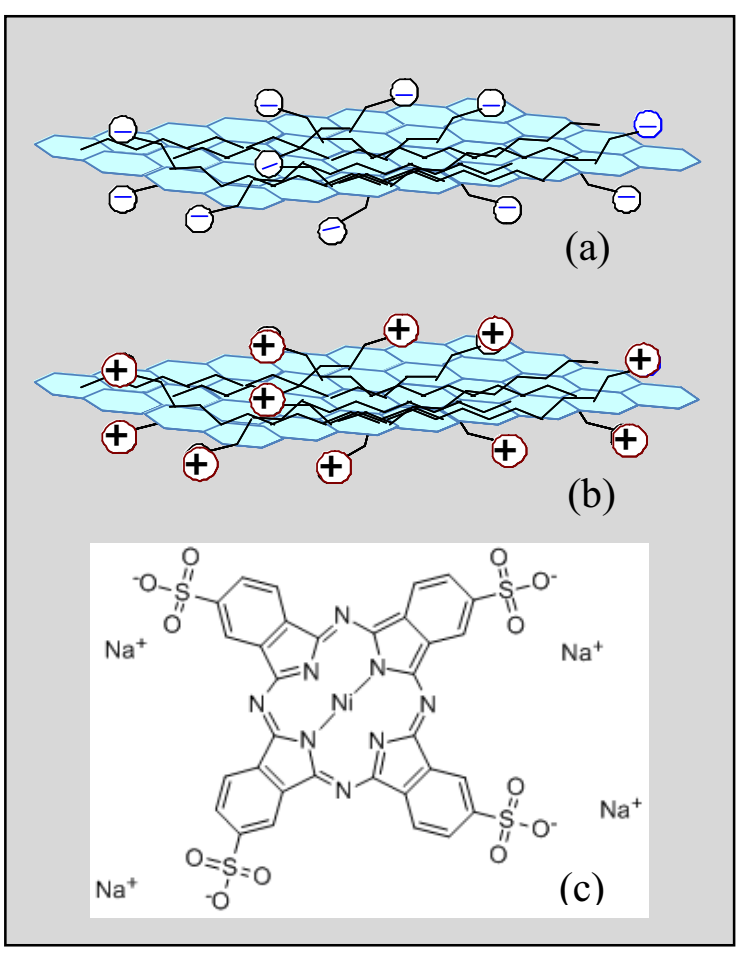

Fig. 1. Schematic representation of graphene-SDS (a) and graphene-CTAB (b). Chemical structure of NiPc derivative used (c).

layer-by-layer (LbL) electrostatic deposition and LB (Langmuir-Blodgett) deposition.

In this study, graphene-based films were deposited onto various substrates (silicon, glass, quartz, and gold interdigitated electrodes (from DropSens) using the electrostatic LbL process). Layers of graphene-CTAB were alternated with polyanions such as PSS, while graphene-SDS layers were alternated with polycations PEI. The surface of glass, quartz, and silicon after cleaning in pirana solution is usually hydrophilic and negatively charged due to the presence of $\mathrm{OH}^{-}$groups, while gold electrodes were additionally treated overnight in $100 \mathrm{mM}$ solution of mercapto-ethylene-sulfonate sodium salt in methanol to enhance the surface negative charge. Samples with the following layer sequence were produced: $(\mathrm{PEI} / \text { graphene-SDS })_{\mathrm{n}}$, where $\mathrm{n}$ is the number of bilayers deposited, typically $n=1,2,3 \ldots$

In the series of graphene-phthalocyanine samples graphene-CTAB was used in alternation with tetrasodium sulphonate nickel phthalocyanine ( $\mathrm{NiPc}-\left(\mathrm{SO}_{3}{ }^{-}\right.$ $\left.\mathrm{Na}^{+}\right)_{4}$ or shortly NiPc) which chemical structure is shown in figure 1c. Electrostatic LbL films were deposited on the above mentioned substrates in the following sequences: $\quad \mathrm{PEI} / \mathrm{PSS} /(\text { graphene-CTAB/NiPc })_{\mathrm{n}}$ or $\mathrm{PEI} /(\mathrm{NiPc} / \text { graphene- CTAB })_{\mathrm{n}}, \mathrm{n}=1,2,3 \ldots$ All chemicals used were purchased form Sigma-Aldrich.

\subsection{Measurements techniques}

The morphology of the LbL films deposited on pieces of Si wafer were studied using AFM (Nanoscope IIIa Digital Technology/Bruker instrument) operating in tapping (scan-assist) mode using Bruker super-sharp tips with a radius of 2-4 $\mathrm{nm}$, and the oscillation frequency of around $100 \mathrm{kHz}$.

Optical absorption spectra of LbL films of graphenephthalocyanine deposited on glass or quartz slides were recorded using Varian Carry 50 spectrophotometer. The thickness and dispersions of refractive index and extinction coefficient of the films produced were studied using J.A. Woollam M2000 spectroscopic ellipsometer. Electrical DC measurements of graphene films deposited on interdigitated gold electrodes from DropSens (containing 100 fringes spaced by $5 \mu \mathrm{m}$ ) were carried out using a Keithley 4200 electrometer in the temperature range from $77^{\circ} \mathrm{K}$ to $393^{\circ} \mathrm{K}$ maintained by Oxford Instrument cryostat Optistat DN2. Gas sensing tests of graphene-phthalocyanine samples deposited on interdigitated electrodes were performed using a $50 \mathrm{ml}$ PTFE cell in which the saturated and 1:10 diluted vapours of $\mathrm{NH}_{3}$ and $\mathrm{HCl}$ were injected with a $10 \mathrm{ml}$ syringe. During such tests, time dependencies of current at fixed DC voltage of $1 \mathrm{~V}$ were recorded using portable UNI-T UT61E multimeter.

\section{Experimental results and discussion}

\subsection{Conductivity of graphene-surfactant composite LbL films}

A typical AFM image of PEI/graphene-SDS film deposited silicon wafer is shown in figure 2. A single flake of about $500 \mathrm{~nm}$ in size, which overlaps with other one or two smaller flakes, is clearly visible. Sectional analysis of this image allowed the estimation of the thickness of a single flake to be about $2 \mathrm{~nm}$.

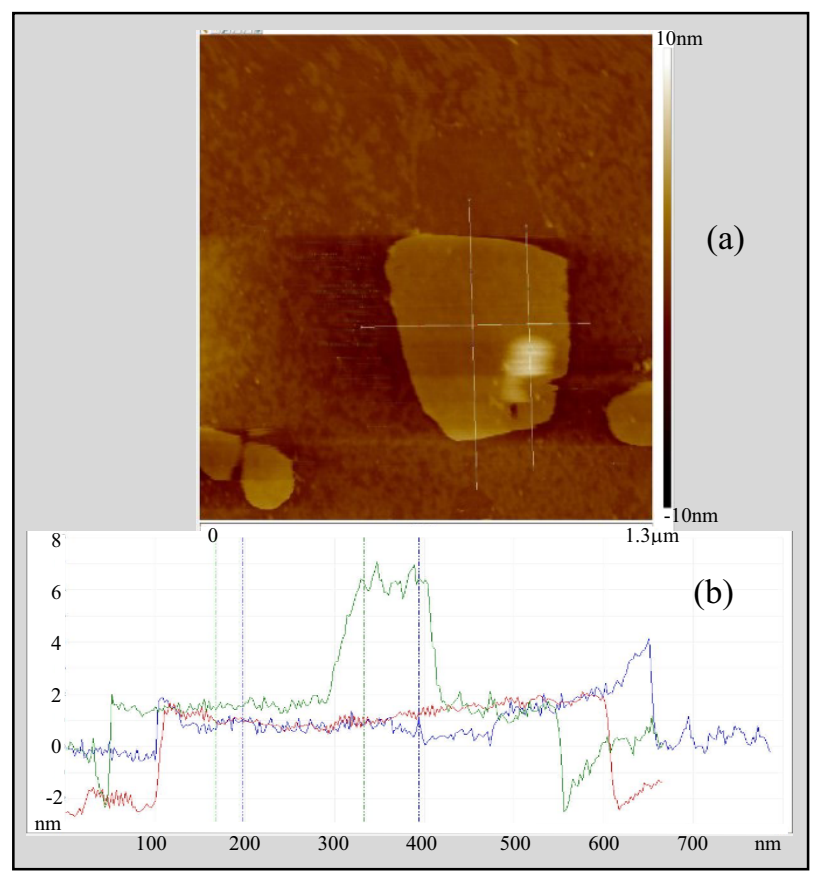

Fig. 2 AFM image of PEI/graphene-SDS layer on Si (a) and its section analysis (b) 
Typical ellipsometry spectra of PEI/graphene-SDS film deposited on silicon are shown in figure 3. Deposition of layers of PEI and graphene-SDS result in substantial shift of $\Delta$ spectra, while $\Psi$ spectra show little changes. This is quite common observation for thin transparent films, where $\Delta$ represent changes in the film thickness while $\Psi$ reflects changes in the refractive index.

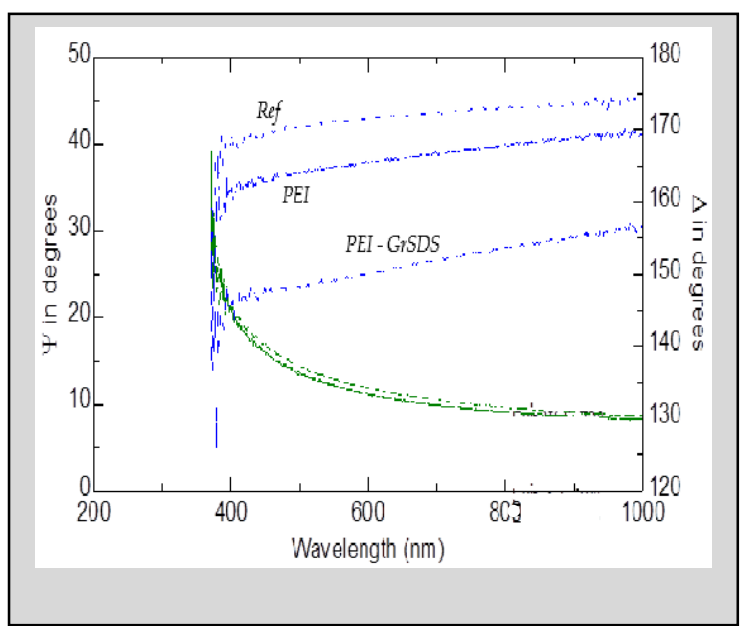

Fig. 3 Spectroscopic ellipsometry data for PEI/graphene-SDS layer on silicon.

Ellipsometry data fitting based on the Lorenz oscillator model for graphene layers [3] yields the thickness increment of $2.5 \mathrm{~nm}$ for PEI/graphene-SDS bilayer which is similar to what was observed directly with AFM.

All graphene-surfactant composite films studied before [3] and recently show Ohmic behaviour as illustrated in figure 4. The dependence of the current against the number of PEI/graphene-SDS bi-layers deposited is nonlinear since the surface coverage in the first couple of layers is less then $100 \%$. Then it stabilises and the conductivity of graphene-surfactant LbL films reaches $0.1 \mathrm{~S} / \mathrm{cm}$ which is appreciable though it is not as high as that for pristine graphene films [4].

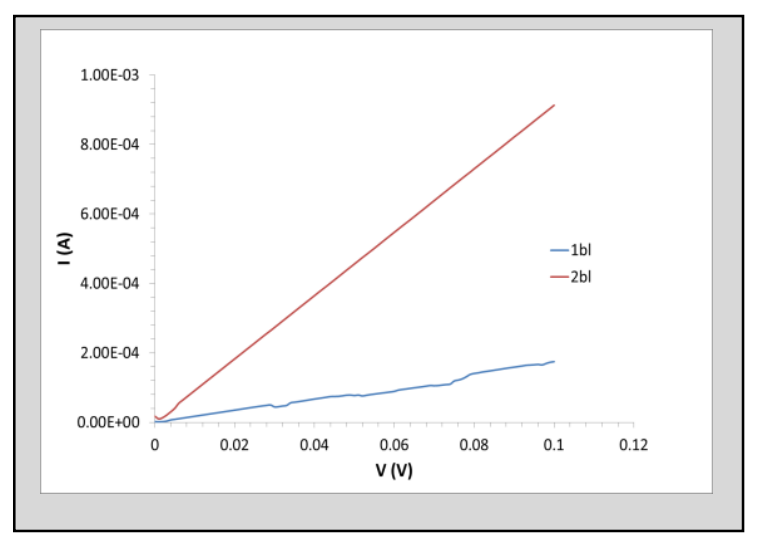

Fig. 4 Typical IV-characteristics of PEI/graphene-SDS films

Obviously, the presence of surfactants physically separates graphene flakes from each other and thus creates potential barriers for conductivity. Subsequently, the mechanism of conductivity must turn into the thermally activated type with conductivity values reduced. Such transformations are apparent form the temperature dependence of conductivity in figure 5 .

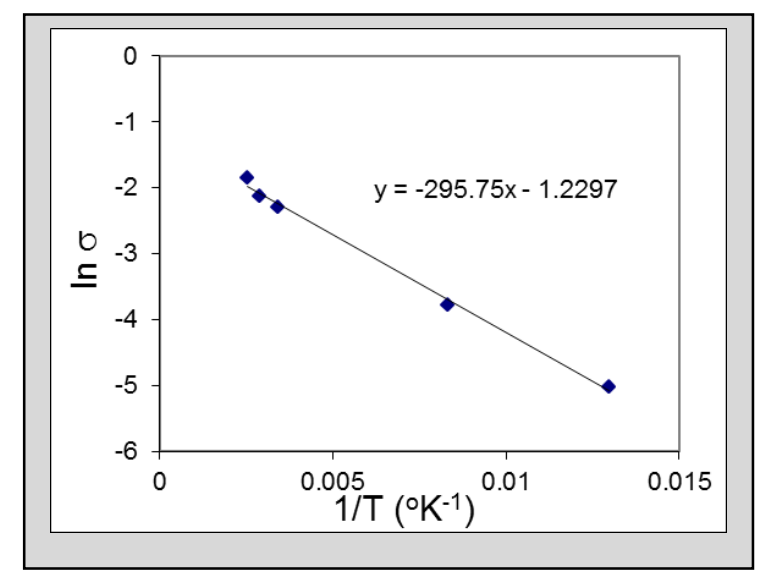

Fig. 5 Temperature dependence of conductivity of $\mathrm{PEI} /$ graphene-SDS films.

The conductivity is clearly of semiconductor type following a general formula $\sigma=\sigma_{0} \exp \left(-E_{a} / k T\right)$. The activation energy $E_{a}$ which could be interpreted as a semiconductor bandgap $E_{g}$ was found to be of $20 \mathrm{meV}$. This is a very small energy comparable with $k T$ at room temperature but it is not zero as compared to pristine graphene [4].

\subsection{Gas sensing properties of graphene-nickel phthalocyanine LbL films}

Composite LbL films of graphene-CTAB alternated with $\mathrm{NiPc}$ were studied with UV-vis absorption spectroscopy, and the results obtained are shown in figure 6 . The main spectral features are typical of NiPc with characteristic spectral absorption bands below 400nm known as the Soret band and the Q-band between 550 and 750nm; the contribution of graphene to the spectra is almost uniform over the whole spectral range and resulted in raising the baseline. However, the presence of graphene has resulted in intriguing transformations of NiPc Q-band. The layers of NiPc deposited either on graphene-CTAB (fig. 6a) or on PEI (fig. 6b) exhibit a Q-band consisting of Qx $(615 \mathrm{~nm})$ and Qy $(670 \mathrm{~nm})$ sub-bands with the Qx peak higher that Qy [8].

(a)

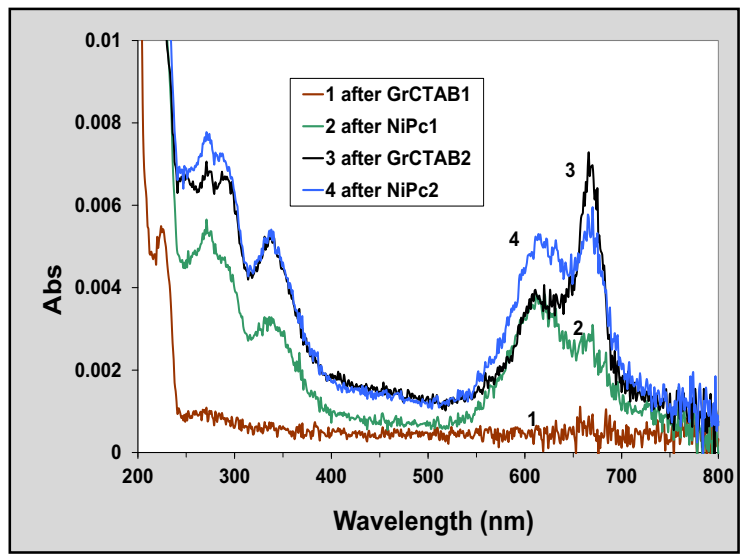


(b)

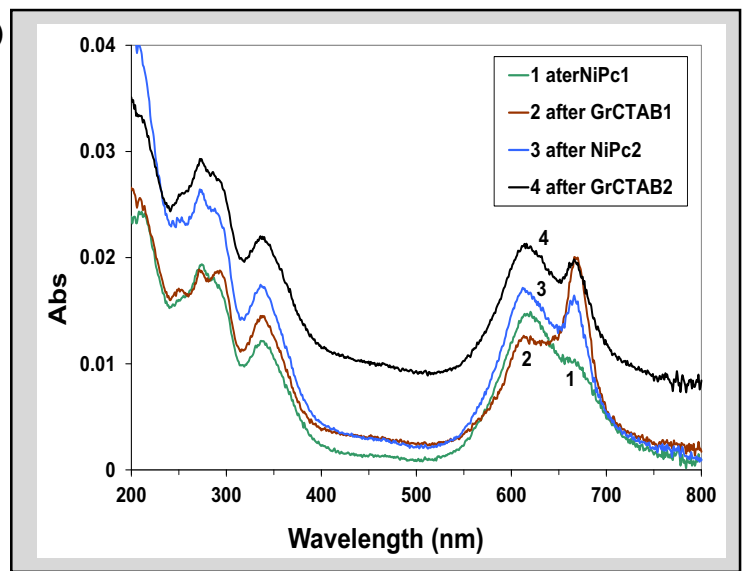

Fig. $6 \mathrm{UV}$-vis absorption spectra of LbL films with the layer sequence of graphene-CTAB/NiPc (a) and NiPc/grapheneCTAB (b)

This is similar to the NiPc spectrum in aqueous solution and typical for insulated NiPc molecules. Deposition of graphene-CTAB on top of NiPc causes a reversal of amplitudes of Qx and Qy with Qy becoming more intense somehow similar to the spectra of metal free phthalocyanines [8]. The observed spectral changes are the indication of interaction of $\pi$-electrons of $\mathrm{NiPc}$ molecules with those of graphene, most-likely resulting in the ionisation of $\mathrm{Ni}$ and subsequent formation of metal-free phthalocyanine molecules $[8,9]$.

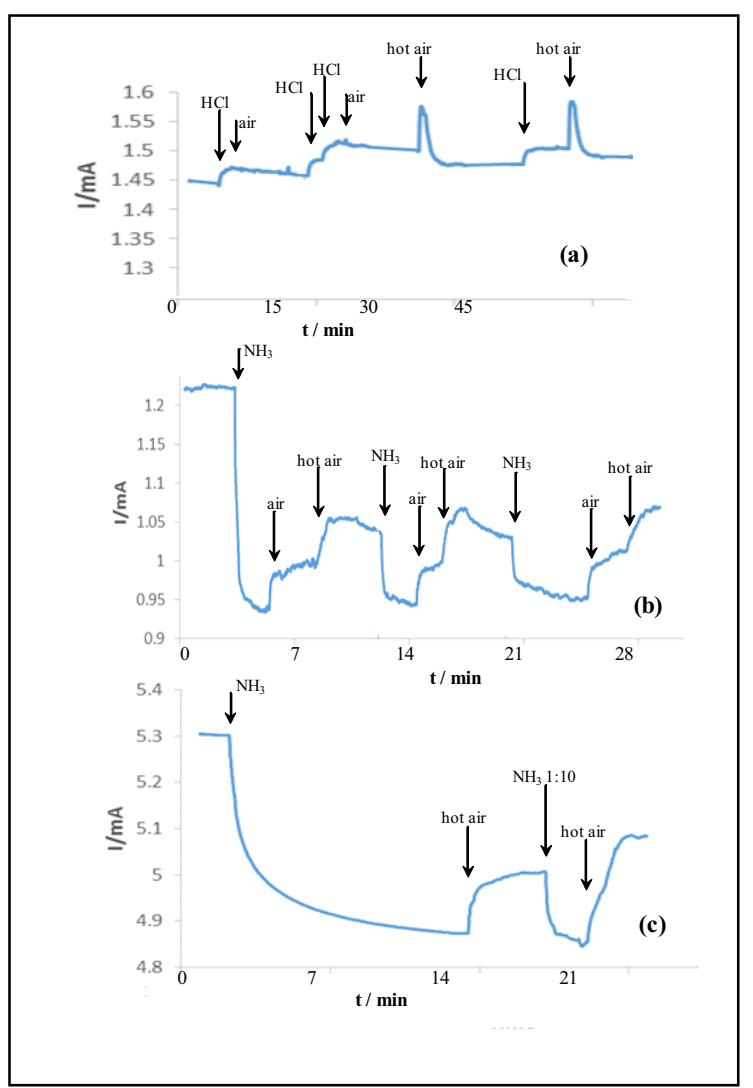

Fig. 7 The responses of conductivity of graphene-CTAB/NiPc films to injection of gases: $\mathrm{HCl}$ (a) and $\mathrm{NH}_{3}(\mathrm{~b}, \mathrm{c})$. Injections of respective vapours, air, and hot air are shown with arrows.
Such a situation (when $\pi$-electrons of phthalocyanine and graphene overlap) is highly desirable for improving gas sensing properties of these composite materials. Indeed, electrical measurements of $\mathrm{NiPc/graphene-CTAB} \mathrm{films}$ confirmed this.

$\mathrm{I}-\mathrm{V}$ characteristics of graphene-CTAB/NiPc LbL films are very similar to those without NiPc. The current recorded at $1 \mathrm{~V}$ DC bias is modulated by injection of vapours of $\mathrm{HCl}$ and $\mathrm{NH}_{3}$ as shown in figure 7. Notably, $\mathrm{HCl}$ saturated vapours caused the conductivity to increase by about $3.5 \%$ (fig. 7a) while saturated $\mathrm{NH}_{3}$ vapours caused its decrease initially by $30 \%$ though following exposures resulted in about $15 \%$ decrease (fig. $7 \mathrm{~b}$ ). The response is fast (just few seconds) but the recovery is much slower and not complete. Additional heating (using hot air) was required to improve the sensor recovery. The sensor response is reproducible (fig. 7b) and dependant on the vapour concentration; 1:10 dilution of saturated $\mathrm{NH}_{3}$ led to much smaller sensor response (fig. 7c).

\section{Conclusions}

The study of electrical characteristics of graphenesurfactant composite LbL films confirmed the thermally activated mode of conductivity typical for semiconductors with the band-gap being about $25 \mathrm{meV}$. Thin LbL films produced by alternation of positively charged layers of graphene-CTAB with negatively charged tetra- sodium-sulphonate NiPc layers demonstrated the interaction of $\pi$-electrons of $\mathrm{NiPc}$ moiety with those of graphene. As a result, the gas sensing properties of such composite materials were improved by combining the high conductivity of graphene with the gas sensing functionality of NiPc. A substantial response of conductivity of graphene thin films functionalized with $\mathrm{NiPc}$ to $\mathrm{HCl}$ and $\mathrm{NH}_{3}$ vapours was observed. Further work is currently underway to study gas sensing properties of graphene-phthalocyanine (or porphyrin) composite films in more detail.

\section{References}

1. S.M. Notley, Langmuir, 28, 4 (2012)

2. $\quad$ N.J. Walch, F. Davis, N. Langford,, J.L. Holmes, S.D. Collyer, S.P.J. Higson, Anal. Chem. 87, 18 (2015)

3. N. J. Walch, A. Nabok, F. Davis, S. P. J. Higson, Beilstein J. Nanotechnol. , 7 (2016)

4. K.S. Novoselov, A.K. Gaim, S.V. Morozov, D. Jiang, Y. Zhang, S.V. Dubonos, I.V. Grigorieva, A.A. Fisov, Science, 306 (2004)

5. E.W. Hill, A. Vijayaragahvan, K. Novoselov, IEEE Sens. J., 11 (2011).

6. S. Basu, P. Bhattacharyya, Sens. Act. B Chem., 173 (2012)

7. P.Leenaerts, B. Partoens, F.M. Peeter, Phys. Rev B, 77 (2008)

8. J. Simon, J.-J. Andre, Molecular Semiconductors, Springler-Verlag, Berlin Heidelberg, 1985

9. D.W. Clack, J.R Yangle, Inorganic Chemistry, 11, 8 (1972) 